\title{
Nonlinear Optimal DTC Design and Stability Analysis for Interior Permanent Magnet Synchronous Motor Drives
}

\author{
Ton Duc Do, Member, IEEE, Han Ho Choi, Member, IEEE, and Jin-Woo Jung, Member, IEEE
}

\begin{abstract}
This paper presents a nonlinear optimal direct torque control (DTC) scheme of interior permanent magnet synchronous motors (IPMSMs) based on an offline approximation approach for electric vehicle (EV) applications. First, the DTC problem is reformulated in the stationary reference frame in order to avoid estimating the stator flux angle, which the previous DTC schemes in the rotating stator reference frame require. Thus, the proposed DTC method eliminates the Park's transformation and consequently it reduces the computational efforts. Particularly, since the estimated stator flux angle is not accurate in low speed range, the proposed method that does not need this information can significantly improve the control performance. Moreover, a nonlinear optimal DTC algorithm is proposed to deal with the nonlinearity of the IPMSM drive system. In this paper, a simple offline $\theta-D$ approximation technique is utilized to appropriately determine the controller gains. Via an IPMSM test-bed with a TI TMS320F28335 DSP, the experimental results demonstrate the feasibility of the proposed DTC method by accomplishing better control performances (e.g., more stable in low speed region, much smaller speed and torque ripples, and faster dynamic responses) compared to the conventional proportionalintegral (PI) DTC scheme under various scenarios with the existence of parameter uncertainties.
\end{abstract}

Index Terms-Direct torque control (DTC), electric vehicle (EV), interior permanent magnet synchronous motor (IPMSM), nonlinear optimal control.

\section{INTRODUCTION}

Electric vehicles (EVs) were invented about two centuries ago. However, the utilization of EVs was stopped in the following century because of both economic and technical aspects such as high cost, capacity shortage of battery, and speed limitation. Recently, the study on the EVs has warmed up as the environmental problems associated with internal combustion engine (ICE) based vehicles become more and more serious. Nowadays, with a fast evolution of battery technology, the eco-friendly EVs become a promising

Manuscript received March 11, 2015; accepted April 17, 2015. This work was supported by the National Research Foundation of Korea (NRF) under Grant 2012R1A2A2A01045312 funded by the Korea government (MSIP, Ministry of Science, ICT \& Future Planning).

The authors are with the Division of Electronics and Electrical Engineering, Dongguk University, Seoul 100-715, Korea (e-mail: jinwjung@ dongguk.edu). alternative to replace the ICE based vehicles [1]- [4].

In EVs, the propulsion system uses an electric motor instead of an ICE in conventional vehicles. There are two main types of electric motors widely utilized in the EVs: induction motor (IM) [5]-[7] and interior permanent magnet synchronous motor (IPMSM) [8]-[10]. In traction applications, the IPMSM is preferable to the IM [11], [12] due to its high efficiency and reliability, high power factor and power density, and high torque to inertia ratio. In addition to these advantages, the invention of highperformance magnets makes the IPMSM a better choice for adjustable-speed motor drives (ASMDs). In general, the control systems for the ASMDs can be categorized into two methods: indirect torque control (ITC) and direct torque control (DTC) [13]. In the ITC, the electromagnetic torque is indirectly regulated through controlling the $q$-axis current; meanwhile, in the DTC, the electromagnetic torque is directly controlled [14]. Therefore, the DTC can achieve a simpler structure and faster dynamic response than the ITC. Consequently, the DTC seems to be more appropriate than the ITC for EV applications.

The DTC concept was introduced about three decades ago by Takahashi and Noguchi for IMs [15]. However, the first DTC papers for IPMSMs were only published until the late 1990's [14], [16]. Although this DTC method possesses some advantages as mentioned above, it still has some disadvantages such as large torque and flux ripples, high acoustic noise, and poor control performance at low speed [17]. To solve these problems, many researches have been conducted and recent publications can be classified into three categories as follows:

1) Schemes that use the hardware [18]-[20] and switching table [21], [22]. The papers in [18], [19] use the matrix converter to reduce the torque and flux ripples. Although the schemes seem to be quite effective, the switching algorithms are complicated. In [20], an analytical approach is proposed to select the hysteresis bands of the DTC to achieve constant switching frequency and low total harmonic distortion (THD) in stator current. However, a field-programmable-gate-array-based control platform is required to avoid the computation delay and then it increases the complexity and cost of the system. 
In [21], [22], novel duty-cycle strategies are presented to reduce both the torque and flux ripples, but the results under parameter uncertainties are not shown.

2) Schemes that use the predictive control algorithms [23], [24]. In [23], although the method is robust to parameter variations, the steady-state speed error is relatively large. The scheme in [24] can reduce the ripple and steadystate error of torque and achieve an acceptable performance at low speed. However, it is not verified that the drive system is insensitive to parameter uncertainties.

3) Schemes that use the space-vector modulation (SVM) to improve the DTC [25]-[28] By incorporating a SVM technique, these DTC schemes can effectively reduce the torque ripple. In addition, the sampling frequency of these SVM-based DTC schemes does not need to be as high as that of the conventional DTC schemes. However, the SVM-based DTC schemes require the coordinate transformation from the stationary reference frame to the rotating reference frame. Meanwhile, the control performance at low speed region may be seriously degraded under parameter variations.

This paper proposes a nonlinear optimal SVM-based DTC scheme of interior permanent magnet synchronous motors (IPMSMs) based on an offline approximation approach for EV applications. By avoiding the flux angle information, the proposed DTC algorithm can not only reduce the computational efforts but also considerably improve the control performance compared with the conventional SVMbased PI DTC scheme. Moreover, the proposed DTC system is designed to effectively deal with the nonlinearity of the IPMSM drive system based on a nonlinear optimal control theory. In this paper, a simple offline $\theta-D$ approximation technique is applied to properly choose the controller gains. Via an IPMSM test-bed with a TI TMS320F28335 DSP, the experimental evidence under a full set of scenarios proves that the proposed DTC scheme is more stable in low speed region, smaller speed and torque ripples, and faster dynamic responses than the conventional SVM-based PI DTC scheme in the presence of parameter uncertainties.

\section{MATHEMatical Model of IPMSMs}

The mathematical equations of a three-phase IPMSM in the stationary reference frame can be expressed as follows:

$$
\left\{\begin{array}{l}
\dot{\omega}=-k_{1} \omega-k_{2}\left(T_{L}-T_{e}\right) \\
\dot{i}_{\alpha}=-k_{3} i_{\alpha}-k_{4} \omega i_{\beta}-k_{5} e_{\alpha}+k_{5} V_{\alpha} \\
\dot{i}_{\beta}=-k_{3} i_{\beta}+k_{4} \omega i_{\alpha}-k_{5} e_{\beta}+k_{5} V_{\beta} \\
e_{\alpha}=-\left[\left(L_{d}-L_{q}\right)\left(\omega i_{d}-\dot{i}_{q}\right)+\omega \lambda_{m}\right] \sin \Theta \\
e_{\beta}=\left[\left(L_{d}-L_{q}\right)\left(\omega i_{d}-\dot{i}_{q}\right)+\omega \lambda_{m}\right] \cos \Theta
\end{array}\right.
$$

where

$$
k_{1}=\frac{B}{J}, k_{2}=\frac{p}{2 J}, k_{3}=\frac{R_{s}}{L_{d}}, k_{4}=1-\frac{L_{q}}{L_{d}}, k_{5}=\frac{1}{L_{d}},
$$

$\omega$ is the electrical rotor speed, $\Theta$ is the electrical rotor position, $i_{\alpha}$ and $i_{\beta}$ are the $\alpha$-axis and $\beta$-axis stator currents in the stationary reference frame, $V_{\alpha}$ and $V_{\beta}$ are the $\alpha$-axis and $\beta$ axis stator voltages, $e_{\alpha}$ and $e_{\beta}$ are $\alpha$-axis and $\beta$-axis extended EMFs, $i_{d}$ and $i_{q}$ are the $d$-axis and $q$-axis stator currents in the synchronously rotating reference frame, $T_{e}$ is the electromagnetic torque, $T_{L}$ is the load torque, $p$ is the number of poles, $R_{s}$ is the stator resistance, $L_{d}$ and $L_{q}$ are the $d$-axis and $q$-axis inductances, $J$ is the rotor inertia, $B$ is the viscous friction coefficient, and $\lambda_{m}$ is the magnet flux linkage.

From [11], [25]-[27], the electromagnetic torque and flux are calculated below:

$$
\left\{\begin{array}{l}
\dot{\lambda}_{\alpha}=V_{\alpha}-R_{s} i_{\alpha} \\
\dot{\lambda}_{\beta}=V_{\beta}-R_{s} i_{\beta} \\
T_{e}=k_{6}\left(\lambda_{\alpha} i_{\beta}-\lambda_{\beta} i_{\alpha}\right) \\
\lambda=\lambda_{s}^{2}=\lambda_{\alpha}^{2}+\lambda_{\beta}^{2}
\end{array}\right.
$$

where $k_{6}=3 p / 4, \lambda_{\alpha}$ and $\lambda_{\beta}$ are the $\alpha$-axis and $\beta$-axis stator flux linkages, $\lambda_{s}$ is the stator flux linkage, and $\lambda$ is the square of the stator flux linkage.

Based on (1) and (2), in the next section, the system model will be transformed to an appropriate form for designing a nonlinear optimal DTC scheme.

\section{Nonlinear Optimal DTC Scheme Design AND STABILITY ANALYSIS}

\section{A. Nonlinear Optimal Controller for Direct Torque Control}

From (2), the first derivatives of $T_{e}$ and $\lambda$ are obtained as follows:

$$
\left\{\begin{aligned}
\dot{T}_{e}= & l_{4} \omega\left(\lambda_{\alpha} i_{\alpha}+\lambda_{\beta} i_{\beta}\right)-k_{3} T_{e}-l_{5}\left(\lambda_{\alpha} e_{\beta}-\lambda_{\beta} e_{\alpha}\right) \\
& +\left(k_{6} i_{\beta}-l_{5} \lambda_{\alpha}\right) V_{\alpha}-\left(k_{6} i_{\alpha}-l_{5} \lambda_{\beta}\right) V_{\beta} \\
\dot{\lambda}= & -2 R_{s}\left(\lambda_{\alpha} i_{\alpha}+\lambda_{\beta} i_{\beta}\right)+2\left(\lambda_{\alpha} V_{\alpha}+\lambda_{\beta} V_{\beta}\right)
\end{aligned}\right.
$$

where $l_{\mathrm{i}}=k_{6} \times k_{i}(i=4,5)$.

Next, let's define new control inputs as

$$
\left\{\begin{array}{l}
u_{1 f b}+u_{1 f f}=\left(k_{6} i_{\beta}-l_{5} \lambda_{\alpha}\right) V_{\alpha}-\left(k_{6} i_{\alpha}-l_{5} \lambda_{\beta}\right) V_{\beta} \\
u_{2 f b}+u_{2 f f}=2 \lambda_{\alpha} V_{\alpha}+2 \lambda_{\beta} V_{\beta}
\end{array}\right.
$$

where $u_{1 f b}$ and $u_{2 f b}$ are the feedback control terms, and $u_{1 f f}$ and $u_{2 f f}$ are the compensating control terms.

Then the following error dynamic equations can be achieved

$$
\begin{gathered}
\dot{x}=f(x)+B u \\
\text { where } x=\left[\begin{array}{lll}
\tilde{\omega} & \tilde{T}_{e} & \tilde{\lambda}
\end{array}\right]^{T}, u=\left[\begin{array}{ll}
u_{1 f b} & u_{2 f b}
\end{array}\right]^{T}, f(x)=A(\tilde{\omega}) x, \\
A(\tilde{\omega})=\left[\begin{array}{ccc}
-k_{1} & k_{2} & 0 \\
0 & -k_{3}+l_{4} \tilde{\omega} & 0 \\
0 & -2 R_{s} & 0
\end{array}\right], \quad B=\left[\begin{array}{ll}
0 & 0 \\
1 & 0 \\
0 & 1
\end{array}\right],
\end{gathered}
$$




$$
\left\{\begin{array}{rl}
u_{1 f f}= & k_{3} T_{\text {eref }}-l_{4} \omega\left[T_{\text {eref }}+\lambda_{\alpha}\left(i_{\alpha}-i_{\beta}\right)+\lambda_{\beta}\left(i_{\alpha}+i_{\beta}\right)\right] \\
& -l_{4} \omega_{\text {ref }} \tilde{T}-l_{5}\left(\lambda_{\alpha} e_{\alpha}-\lambda_{\beta} \lambda_{\beta}\right) \\
u_{2 f f}= & 2 R_{s}\left[T_{\text {eref }}+\lambda_{\alpha}\left(i_{\alpha}-i_{\beta}\right)+\lambda_{\beta}\left(i_{\alpha}+i_{\beta}\right)\right]
\end{array},\right.
$$

and $\omega_{\text {ref }}$ is the speed reference, $T_{\text {eref }}=T_{L}+\omega_{\text {ref }} \cdot k_{1} / k_{2}$ is the torque reference, $\tilde{\omega}=\omega-\omega_{\text {ref }}$ is the speed error, $\widetilde{T}_{e}=T_{e}-T_{\text {eref }}$ is the torque error, and $\tilde{\lambda}=\lambda-\lambda_{\text {ref }}$ is the flux error. Note that $\lambda_{\text {ref }}$ is the flux reference calculated from the torque reference $T_{\text {eref }}$ through the maximum torque per ampere (MTPA) algorithm.

According to nonlinear control theories [29], [30], we need to find the stabilizing control term $u$ that minimizes the following cost function for the system (5):

$$
J=\frac{1}{2} \int_{0}^{\infty}\left(x^{T} Q x+u^{T} R u\right) d t .
$$

Then, the nonlinear optimal controller can be obtained by solving the following Hamilton-Jacobi-Bellman (HJB) partial differential equation:

$$
\frac{\partial V^{T}}{\partial x} f(x)-\frac{1}{2} \frac{\partial V^{T}}{\partial x} B R^{-1} B^{T} \frac{\partial V}{\partial x}+\frac{1}{2} x^{T} Q x=0 .
$$

Assuming that $V(x)$ is positive and continuously differentiable with $V(0)=0$, then the control law is given by

$$
u=-R^{-1} B^{T} \frac{\partial V}{\partial x} .
$$

However, the well-known HJB equation (7) is extremely difficult to solve. In [29], [30], an approximation technique called the $\theta-D$ method is proposed to efficiently achieve the solution of the HJB equation (7) for a specified class of nonlinear systems.

First, the weighting matrix $Q$ and $\partial V / \partial x$ are rewritten as

$$
Q=Q_{0}+\sum_{i=1}^{\infty} D_{i} \theta^{i} ; \quad \frac{\partial V}{\partial x}=\sum_{i=0}^{\infty} T_{i} \theta^{i} x
$$

where $Q_{0} \in R^{3 \times 3}$ and $D_{i} \in R^{3 \times 3}$ are a symmetric constant and symmetric state-dependent matrix, respectively, $\theta$ is a scalar, $T_{i}$ are symmetric matrices, and $i$ is an integer. Note that $\theta$ and $D_{i}$ are chosen such that $Q$ is symmetric semi-positive definite.

Substituting (9) into (7) then equating the coefficients of the powers of $\theta$ to zero, the following equations are achieved

$$
\begin{gathered}
T_{0} A_{0}+A_{0}^{T} T_{0}-T_{0} B R^{-1} B^{T} T_{0}+Q_{0}=0 \\
T_{1} A_{1}+A_{1}^{T} T_{1}+\frac{T_{0} \Delta A}{\theta}+\frac{\Delta A^{T} T_{0}}{\theta}+D_{1}=0 \\
\vdots \\
T_{n} A_{1}+A_{1}^{T} T_{n}+\frac{T_{n-1} \Delta A}{\theta}+\frac{\Delta A^{T} T_{n-1}}{\theta}-\sum_{i=1}^{n-1} T_{i} B R^{-1} B^{T} T_{n-i}+D_{n}=0
\end{gathered}
$$

where $A_{1}=A_{0}-B R^{-1} B^{T} T_{0}$ and

$$
A_{0}=\left[\begin{array}{ccc}
-k_{1} & k_{2} & 0 \\
0 & -k_{3} & 0 \\
0 & -2 R_{s} & 0
\end{array}\right], \quad \Delta A=\left[\begin{array}{ccc}
0 & 0 & 0 \\
0 & l_{4} \tilde{\omega} & 0 \\
0 & 0 & 0
\end{array}\right] .
$$

It is clear that (10) is an algebraic Riccati equation (ARE) while (11) and (12) are state-dependent Lyapunov equations (SDLEs). In order to transform (11) and (12) to the AREs, let us set the matrices $D_{i}$ and $T_{i}$ as

$$
\begin{aligned}
& D_{i}=-m_{i} e^{-n_{i} t}\left(\frac{T_{i-1} \Delta A}{\theta}+\frac{\Delta A^{T} T_{i-1}}{\theta}-\sum_{j=1}^{i-1} T_{j} B R^{-1} B^{T} T_{i-j}\right) \\
& T_{i}=\frac{\bar{T}_{i}}{\theta^{i}}
\end{aligned}
$$

where $m_{i}$ and $n_{i}$ are positive numbers, and $\bar{T}_{i}$ are symmetric matrices. It should be noted that $\bar{T}_{0}=T_{0}$. Then the SDLEs (11) and (12) are transformed into

$$
\begin{gathered}
\bar{T}_{1} A_{1}+A_{1}^{T} \bar{T}_{1}+\left(T_{0} \Delta A+\Delta A^{T} T_{0}\right) \varepsilon_{1}=0 \\
\vdots \\
\bar{T}_{n} A_{1}+A_{1}^{T} \bar{T}_{n}+\left(\bar{T}_{n-1} \Delta A+\Delta A^{T} \bar{T}_{n-1}-\sum_{i=1}^{n-1} \bar{T}_{i} B R^{-1} B^{T} \bar{T}_{n-i}\right) \varepsilon_{n}=0
\end{gathered}
$$

where $\varepsilon_{i}=1-m_{i} e^{-n_{i} t}$.

According to [29], [30], the SDLEs (14) and (15) can be solved by the Kronecker product. However, by establishing $\bar{T}_{i}=T_{i}^{C} \varepsilon_{i} \tilde{\omega}^{i}$ and $\Delta A=\tilde{\omega} \Delta A_{C}$, (14) and (15) can be further simplified to the following algebraic Lyapunov equations [31]:

$$
\begin{gathered}
T_{1}^{C} A_{1}+A_{1}^{T} T_{1}^{C}+T_{0} \Delta A_{C}+\Delta A_{C}^{T} T_{0}=0 \\
\vdots \\
T_{i}^{C} A_{1}+A_{1}^{T} T_{i}^{C}+T_{i-1}^{C} \Delta A_{C}+\Delta A_{C}^{T} T_{i-1}^{C}-\sum_{k=1}^{i-1} T_{k}^{C} B R^{-1} B^{T} T_{i-k}^{C}=0
\end{gathered}
$$

where $T_{i}^{C}$ are symmetric matrices and $\Delta A_{C}=\left[\begin{array}{ccc}0 & 0 & 0 \\ 0 & l_{4} & 0 \\ 0 & 0 & 0\end{array}\right]$.

Then, the approximate control law is given by

$$
u=-R^{-1} B^{T} \sum_{i=0}^{N} T_{i}^{C} \varepsilon_{i} \tilde{\omega}^{i} x
$$

where $T_{0}{ }^{C}=\bar{T}_{0}=T_{0}, \varepsilon_{0}=1$, and $N$ is the number of series calculated offline. Note that the value of $N$ is chosen via extensive simulation and experimental studies as done in the state-dependent Riccati equation methods in [32] and [33].

By substituting the control law (18) and the compensating terms (5) into (4), the $\alpha$-axis and $\beta$-axis stator voltages can be expressed as 


$$
\left\{\begin{array}{l}
V_{\alpha}=\frac{u_{1} \times 2 \lambda_{\beta}+u_{2} \times\left(k_{6} i_{\alpha}-l_{5} \lambda_{\beta}\right)}{\left(k_{6} i_{\beta}-l_{5} \lambda_{\alpha}\right) \times 2 \lambda_{\beta}+2 \lambda_{\alpha} \times\left(k_{6} i_{\alpha}-l_{5} \lambda_{\beta}\right)} \\
V_{\beta}=\frac{u_{2} \times\left(k_{6} i_{\beta}-l_{5} \lambda_{\alpha}\right)-u_{1} \times 2 \lambda_{\alpha}}{\left(k_{6} i_{\beta}-l_{5} \lambda_{\alpha}\right) \times 2 \lambda_{\beta}+2 \lambda_{\alpha} \times\left(k_{6} i_{\alpha}-l_{5} \lambda_{\beta}\right)}
\end{array} .\right.
$$

where $u_{l}=u_{1 f b}+u_{1 f f}$ and $u_{2}=u_{2 f b}+u_{2 f f}$.

\section{B. Stability Analysis}

Referring to [29], both Lemma and Theorem can be given as follows:

Lemma 1: The series $\sum_{i=0}^{\infty} T_{i} \theta^{i}$ is pointwise convergent and positive definite.

Proof: This Lemma can be proven in a similar way to [29].

Theorem 1: The closed-loop control system obtained by the error dynamics (5) and the nonlinear feedback control law (18) is semi-globally asymptotically stable.

Proof: First, let us define the following Lyapunov function:

$$
L(x)=\frac{1}{2} x^{T} \sum_{i=0}^{\infty} \bar{T}_{i} x .
$$

From Lemma 1, $\sum_{i=0}^{\infty} \bar{T}_{i}$ is positive definite, so $L(x)>0$. Thus, its time derivative can be obtained as

$$
\begin{aligned}
\frac{d L(x)}{d t} & =\left[\frac{\partial L(x)}{\partial t}\right]^{T} \dot{x}=\left[\frac{\partial L(x)}{\partial t}\right]^{T}[f(x)+B u] \\
& =\left[x^{T} \sum_{i=0}^{\infty} \bar{T}_{i}+\frac{1}{2} x^{T} \sum_{i=0}^{\infty} \frac{\partial \bar{T}_{i}}{\partial x} x\right][f(x)+B u] .
\end{aligned}
$$

Besides, $V_{x}=\sum_{i=0}^{\infty} \bar{T}_{i} x\left(V_{x}=\partial V / \partial x\right)$ satisfies the following HJB equation:

$$
V_{x}^{T}[f+B u]+\frac{1}{2} u^{T} R u+\frac{1}{2} x^{T}\left(Q_{0}+\sum_{i=1}^{\infty} D_{i} \theta^{i}\right) x=0 .
$$

Then, (22) is equivalent to

$$
V_{x}^{T}[f+B u]=-\frac{1}{2} u^{T} R u-\frac{1}{2} x^{T}\left(Q_{0}+\sum_{i=1}^{\infty} D_{i} \theta^{i}\right) x .
$$

Therefore, substituting (23) into (21) yields the following equation:

$$
\begin{aligned}
\frac{d L(x)}{d t}= & -\frac{1}{2} u^{T} R u-\frac{1}{2} x^{T}\left(Q_{0}+\sum_{i=1}^{\infty} D_{i} \theta^{i}\right) x \\
& +\frac{1}{2} x^{T} \sum_{i=0}^{\infty} \frac{\partial \bar{T}_{i}}{\partial x} x[f+B u]
\end{aligned}
$$

Since $u=-R^{-1} B^{T} \sum_{i=0}^{\infty} \bar{T}_{i} x$, the following equation can be established:

$$
\begin{aligned}
& -\frac{1}{2} u^{T} R u-\frac{1}{2} x^{T}\left(Q_{0}+\sum_{i=1}^{\infty} D_{i} \theta^{i}\right) x \\
& =-\frac{1}{2} x^{T}\left[Q_{0}+\sum_{i=0}^{\infty} \bar{T}_{i} B R^{-1} B^{T} \sum_{i=0}^{\infty} \bar{T}_{i}+\sum_{i=1}^{\infty} D_{i} \theta^{i}\right] x .
\end{aligned}
$$

Applying Courant-Fischer theorem, we have

$$
\begin{aligned}
& -\frac{1}{2} x^{T}\left[Q_{0}+\sum_{i=0}^{\infty} \bar{T}_{i} B R^{-1} B^{T} \sum_{i=0}^{\infty} \bar{T}_{i}+\sum_{i=1}^{\infty} D_{i} \theta^{i}\right] x \\
& \leq-\frac{1}{2} \lambda_{\min }\left[Q_{0}+\sum_{i=0}^{\infty} \bar{T}_{i} B R^{-1} B^{T} \sum_{i=0}^{\infty} \bar{T}_{i}+\sum_{i=1}^{\infty} D_{i} \theta^{i}\right]\|x\|^{2}
\end{aligned}
$$

where $\lambda_{\min }$ is the minimum eigenvalue of the matrix in the square bracket in (26).

Thus, the following inequality can be given:

$$
\begin{gathered}
\frac{d L(x)}{d t} \leq \frac{1}{2} x^{T} \sum_{i=0}^{\infty} \frac{\partial \overline{T_{i}}}{\partial x} x[f+B u] \\
-\frac{1}{2} \lambda_{\min }\left[Q_{0}+\sum_{i=0}^{\infty} \bar{T}_{i} B R^{-1} B^{T} \sum_{i=0}^{\infty} \bar{T}_{i}+\sum_{i=1}^{\infty} D_{i} \theta^{i}\right]\|x\|^{2} \\
\leq-\frac{1}{2} C_{\lambda}\|x\|^{2}+\frac{1}{2}\|x\|^{2}\left\|\sum_{i=0}^{\infty} \frac{\partial \bar{T}_{i}}{\partial x} x\right\|\left\|A-B R^{-1} B^{T} \sum_{i=0}^{\infty} \bar{T}_{i}\right\|
\end{gathered}
$$

where

$$
C_{\lambda}=\lambda_{\min }\left[Q_{0}+\sum_{i=0}^{\infty} \bar{T}_{i} B R^{-1} B^{T} \sum_{i=0}^{\infty} \bar{T}_{i}+\sum_{i=1}^{\infty} D_{i} \theta^{i}\right]>0 .
$$

A sufficient small $\varepsilon_{i}$ is chosen to satisfy the following inequality:

$$
C_{\lambda}>\left\|\sum_{i=0}^{\infty} \frac{\partial \bar{T}_{i}}{\partial x} x\right\|\left\|A-B R^{-1} B^{T} \sum_{i=0}^{\infty} \bar{T}_{i}\right\| .
$$

Finally, the inequality $d L(x) / d t<0$ is also satisfied. Hence, the closed-loop system is semi-globally asymptotically stable.

\section{Robustness with respect to Parameter Variations}

It is well-known that the optimal regulator minimizing the quadratic performance cost function (6) for a linear system usually guarantees robust performances with minimum $-6 \mathrm{~dB}$ gain margin and $60^{\circ}$ phase margin.

Similarly, the proposed control law guarantees the robustness to model parameter variations. Consider the following perturbed model

$$
\dot{x}=f(x)+\Delta f(x)+B u .
$$

Then, the time derivative of the Lyapunov function (20) can be rewritten as 


$$
\begin{aligned}
\frac{d L(x)}{d t}= & {\left[\frac{\partial L(x)}{\partial t}\right]^{T} \dot{x}=\left[\frac{\partial L(x)}{\partial t}\right]^{T}[f(x)+\Delta f(x)+B u] } \\
= & {\left[x^{T} \sum_{i=0}^{\infty} \bar{T}_{i}+\frac{1}{2} x^{T} \sum_{i=0}^{\infty} \frac{\partial \bar{T}_{i}}{\partial x} x\right][f(x)+\Delta f(x)+B u] } \\
= & {\left[x^{T} \sum_{i=0}^{\infty} \bar{T}_{i}+\frac{1}{2} x^{T} \sum_{i=0}^{\infty} \frac{\partial \bar{T}_{i}}{\partial x} x\right] \Delta f(x) } \\
& -\frac{1}{2} x^{T}\left[Q_{0}+\sum_{i=0}^{\infty} \bar{T}_{i} B R^{-1} B^{T} \sum_{i=0}^{\infty} \bar{T}_{i}+\sum_{i=1}^{\infty} D_{i} \theta^{i}\right] x \\
& +\frac{1}{2} x^{T} \sum_{i=0}^{\infty} \frac{\partial \bar{T}_{i}}{\partial x} x[f+B u] .
\end{aligned}
$$

If the perturbation $\Delta f(x)$ satisfies the following inequality

$$
\begin{aligned}
& {\left[x^{T} \sum_{i=0}^{\infty} \bar{T}_{i}+\frac{1}{2} x^{T} \sum_{i=0}^{\infty} \frac{\partial \bar{T}_{i}}{\partial x} x\right] \Delta f(x)<} \\
& \frac{1}{2} x^{T}\left[Q_{0}+\sum_{i=0}^{\infty} \bar{T}_{i} B R^{-1} B^{T} \sum_{i=0}^{\infty} \bar{T}_{i}+\sum_{i=1}^{\infty} D_{i} \theta^{i}\right] x \\
& -\frac{1}{2} x^{T} \sum_{i=0}^{\infty} \frac{\partial \bar{T}_{i}}{\partial x} x[f+B u],
\end{aligned}
$$

then $\dot{L}(x)<0$ for all nonzero $x$. This implies that the proposed method can tolerate any perturbation $\Delta f(x)$ satisfying the above inequality (31). It should be noted that we can choose a sufficient small $\varepsilon_{i}$ to make the inequality (31) feasible.

\section{Stability Analysis Considering Estimation Errors on Torque and Flux}

In Theorem 1, it is assumed that the flux and torque are available. However, in reality, they can be only estimated and then there exist some errors between the actual value and the estimated value. Consequently, the control law (18) should be replaced by the following:

$$
u_{a}=-R^{-1} B^{T} \sum_{i=0}^{N} T_{i}^{C} \varepsilon_{i} \tilde{\omega}^{i} \hat{x}
$$

where $\hat{x}$ is an estimation of $x$. Denote the estimation error as

$$
d=x-\hat{x} \text {. }
$$

If the estimation error is not so large, we can choose a positive constant $\kappa$ so that $\|d\| \leq \kappa$. By referring to the proof of Theorem 1, we can show that

$$
\begin{aligned}
\frac{d L(x)}{d t}=\left[\frac{\partial L(x)}{\partial t}\right]^{T} \dot{x}=\left[\frac{\partial L(x)}{\partial t}\right]^{T}\left[f(x)+B u_{a}\right] \\
=\left[x^{T} \sum_{i=0}^{\infty} \bar{T}_{i}+\frac{1}{2} x^{T} \sum_{i=0}^{\infty} \frac{\partial \bar{T}_{i}}{\partial x} x\right]\left[f(x)+B u+B\left(u_{a}-u\right)\right] \\
\leq-\frac{1}{2} C_{\lambda}\|x\|^{2}+\frac{1}{2}\|x\|^{2}\left\|\sum_{i=0}^{\infty} \frac{\partial \bar{T}_{i}}{\partial x} x\right\|\left\|A-B R^{-1} B^{T} \sum_{i=0}^{\infty} \bar{T}_{i}\right\| \\
+\kappa \mid\|x\|\left[\left\|\sum_{i=0}^{\infty} \bar{T}_{i}\right\|+\frac{1}{2}\left\|\sum_{i=0}^{\infty} \frac{\partial \bar{T}_{i}}{\partial x}\right\|\|x\|\right] \\
\leq-\frac{1}{2}\left(C_{\lambda 1}-\kappa\left\|\sum_{i=0}^{\infty} \frac{\partial \bar{T}_{i}}{\partial x}\right\|\|\|^{2}+\kappa\|x\|\left\|_{i=0}^{\infty} \bar{T}_{i}\right\|\right.
\end{aligned}
$$

where $C_{\lambda 1}=C_{\lambda}-\left\|\sum_{i=0}^{\infty} \frac{\partial \bar{T}_{i}}{\partial x} x\right\|\left\|A-B R^{-1} B^{T} \sum_{i=0}^{\infty} \bar{T}_{i}\right\|$.

Also, we can choose a sufficient small $\varepsilon_{i}$ so that $C_{\lambda 1}>0$. If the estimation error satisfies $\|x-\hat{x}\|=\kappa<C_{\lambda 1} /\left\|\sum_{i=0}^{\infty} \frac{\partial \bar{T}_{i}}{\partial x}\right\|$, then the above inequality implies that the set $\Omega=\{x:\|x\| \leq \mu\}$ is invariant, where $\mu=2 \kappa\left\|\sum_{i=0}^{\infty} \bar{T}_{i}\right\| /\left(C_{\lambda 1}-\kappa\left\|\sum_{i=0}^{\infty} \frac{\partial \bar{T}_{i}}{\partial x}\right\|\right)$. After all, it can be seen that the closed-loop system response is uniformly ultimately bounded.

\section{MTPA TRAJECTORY TRACKING, LOAD TORQUE Calculation, and Design Procedure}

This section discusses the three following issues: the MTPA trajectory tracking, load torque calculation, and gain tuning procedure. Also, the overall diagrams of the proposed SVM-based DTC scheme and conventional SVM-based PI DTC scheme are analyzed and compared.

\section{A. Maximum Torque per Ampere Trajectory Tracking}

In this subsection, the procedure to calculate $\lambda_{\text {ref }}$ from $T_{\text {eref }}$ is elaborated. According to [34], [35], the maximum torque per ampere (MTPA) trajectory can be obtained if the relation between the $d$-axis current $i_{d}$ and $q$-axis current $i_{q}$ satisfies the following equation:

$$
i_{d}=k_{7} i_{q}^{2}
$$

where $k_{7}=\left(L_{d}-L_{q}\right) / \lambda_{m}$. It is noted that (35) is an approximated equation which is widely utilized in several papers with an acceptable tolerance [34], [35]. However, the equation (35) cannot be directly used because the state variables in DTC are $\lambda$ and $T_{e}$. Therefore, (35) is used to find the relation between $\lambda$ and $T_{e}$.

Alternatively, the electromagnetic torque and stator flux can be calculated by: 


$$
\left\{\begin{array}{l}
\lambda_{d}=L_{d} i_{d}+\lambda_{m} \\
\lambda_{q}=L_{q} i_{q} \\
T_{e}=k_{8} \lambda_{d} \lambda_{q}+k_{9} \lambda_{q} \\
\lambda=\lambda_{d}^{2}+\lambda_{q}^{2}
\end{array}\right.
$$

where

$$
k_{8}=\frac{3 p}{4}\left(\frac{1}{L_{q}}-\frac{1}{L_{d}}\right), k_{9}=\frac{3 p}{4} \frac{\lambda_{m}}{L_{d}} .
$$

Using (35) and (36), the following relation can be achieved

$$
\left\{\begin{array}{l}
\lambda_{d}=\frac{k_{7} k_{11}^{2}}{k_{10}} \lambda_{q}^{2}+\lambda_{m} \\
T_{e}=\frac{k_{8} k_{7} k_{11}^{2}}{k_{10}} \lambda_{q}^{3}+\left(k_{8} \lambda_{m}+k_{9}\right) \lambda_{q}
\end{array}\right.
$$

where $k_{10}=1 / L_{d}$ and $k_{11}=1 / L_{q}$.

In summary, the procedure to calculate $\lambda_{\text {ref }}$ from $T_{\text {eref }}$ via MTPA trajectory tracking is as follows: Given the value of $T_{\text {eref, }}$, by solving the third-order polynomial in the second equation of (37), $\lambda_{q}$ is obtained. Then $\lambda_{d}$ is calculated by the first equation of (37). Finally, $\lambda_{\text {ref }}$ is obtained from the fourth equation of (36). It should be noted that with defined $k_{i}$, the second equation of (37) has only one real root for $\lambda_{q}$. Therefore, substituting the solution $\left(\lambda_{q}, \lambda_{d}\right)$ calculated from (37) into (36) yields only one solution of $\left(i_{q}, i_{d}\right)$.

Remark 1: The solution for the third-degree polynomial in the last equation of (37) can be easily calculated by the following formula:

$\lambda_{q}=\sqrt[3]{-\frac{c}{2 a}+\sqrt{\left(\frac{c}{2 a}\right)^{2}+\left(\frac{b}{3 a}\right)^{3}}}+\sqrt[3]{-\frac{c}{2 a}-\sqrt{\left(\frac{c}{2 a}\right)^{2}+\left(\frac{b}{3 a}\right)^{3}}}$

where $a=k_{8} k_{7} k_{11}{ }^{2} / k_{10}, b=\left(k_{8} \lambda_{m}+k_{9}\right)$, and $c=-T_{e}$. Note that the previous DTC schemes use a look-up table for an MTPA trajectory tracking [11], [25]-[27]. Although these methods are quite simple, a high-degree of accuracy is not provided. By using the equation (38), the MTPA trajectory is online tracked with much higher accuracy. In addition, with the high-speed TI DSP TMS320F28335 used in this paper, there is no difficult to implement the overall control system that includes the compensating terms (5), feedback control terms (18), and MTPA trajectory tracking (38).

\section{B. Load Torque Estimation}

In (5), it can be seen that the load torque $\left(T_{L}\right)$ information is needed to calculate the torque reference $T_{\text {eref }}$. In most recent papers, the load torque is estimated by an observer. Although this approach can accurately estimate the load torque, it complicates the control schemes. Alternatively, the load torque can be simply obtained from the first equation of (1).

First, the rotor angular acceleration $\beta$ can be derived from [36]-[38]:

$$
\hat{\beta}(k)=\frac{\varphi}{T_{s}+\varphi} \hat{\beta}(k-1)+\frac{1}{T_{s}+\varphi}[\omega(k)-\omega(k-1)]
$$

where $k$ and $k-1$ denote two consecutive sampling instants, $T_{\mathrm{s}}$ is the sampling period, and $\varphi$ is a sufficiently small time constant.

Then, based on the first equation of (1), the load torque is calculated by the following equation:

$$
\hat{T}_{L}(k)=-\frac{k_{1}}{k_{2}} \omega(k)+T_{e}(k)-\frac{1}{k_{2}} \hat{\beta}(k) .
$$

\section{Gain Tuning Procedure}

The control gains $T_{i}^{C}$ are tuned via adjusting the weighting matrices $Q_{0}$ and $R$. Besides, $\varepsilon_{i}$ are tuned by changing the design parameters $k_{i}$ and $l_{i}$. That is, the gains of the proposed nonlinear optimal controller are tuned by the following steps:

1) Assume that $\varepsilon_{i}=1$. Then tune $Q_{0}, R$, and $N$ by the rule in [39], [33] to obtain the satisfactory control performance.

2) With the above $Q_{0}$ and $R$, select $m_{i}$ and $n_{i}$ by the method in [29], [30] to improve the control performance.

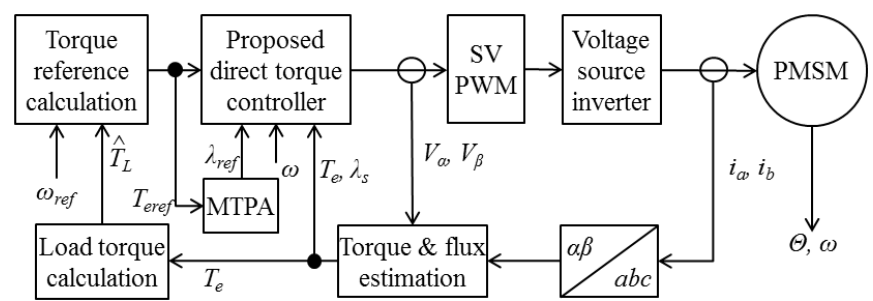

Fig. 1. Overall block diagram of the proposed nonlinear optimal SVM-based DTC scheme.

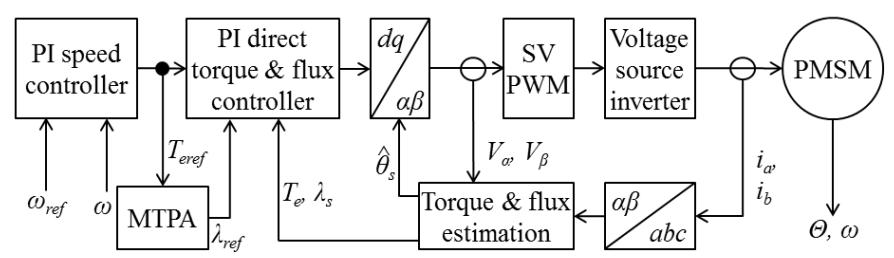

Fig. 2. Overall block diagram of the conventional SVM-based PI DTC scheme.

Fig. 1 shows the overall block diagram of the proposed nonlinear optimal SVM-based DTC scheme. For comparison, Fig. 2 depicts the block diagram of the conventional PI DTC scheme [11], [25]-[27]. As seen in Fig. 1, the proposed DTC system is designed in the stationary reference frame in order to avoid estimating the stator flux angle that is used by previous SVM-based DTC schemes in the synchronously rotating reference frame. Notice that the control performance is severely deteriorated because the estimated stator flux angle is not accurate in low speed region. Consequently, the proposed DTC technique can at once decrease the computational efforts and enhance the control performance in comparison with the conventional SVM-based PI DTC scheme illustrated in Fig. 2. 


\section{EXPERIMENTAL VERIFICATIONS}

\section{A. Experimental Setup, Gain Selection, and Conditions}

This section experimentally investigates the feasibility and effectiveness of the proposed SVM-based DTC scheme through various scenarios. Table I tabulates the parameters of a three-phase prototype IPMSM drive system used in experiments. Also, the proposed algorithm is implemented on a control board with a Texas Instruments TMS320F28335 DSP. Considering the system efficiency and control performance, the sampling frequency $\left(T_{s}\right)$ and PWM frequency $\left(f_{s}\right)$ are chosen at $200 \mu$ s and $5 \mathrm{kHz}$, respectively. Fig. 3 presents the experimental setup of the prototype IPMSM drive system. It should be noted that an electric brake is used to generate the load torque. By using a programmable dc power supply, the input current to the brake is controlled. As the load torque generated is proportional to the input dc current of the brake, the load torque can be flexibly applied via controlling the input dc current.

TABLE I

PARAMETERS OF A PROTOTYPE IPMSM DRIVE

PARAMETERS OF A PROTOTYPE IPMSM DRIVE
\begin{tabular}{|c|c|c|c|}
\hline Symbol & Parameter & Value & Unit \\
\hline$P_{\text {rated }}$ & Rated power & 390 & $\mathrm{~W}$ \\
\hline$p$ & Number of poles & 4 & - \\
\hline$R_{s}$ & Stator resistance & 2.48 & $\Omega$ \\
\hline$L_{q}$ & $q$-axis inductance & 113.91 & $\mathrm{mH}$ \\
\hline$L_{d}$ & $d$-axis inductance & 74.98 & $\mathrm{mH}$ \\
\hline$\lambda_{m}$ & Magnet flux linkage & 0.193 & $\mathrm{~Wb}$ \\
\hline$J$ & Equivalent rotor inertia & 0.00042 & $\mathrm{~kg} \cdot \mathrm{m}^{2}$ \\
\hline$B$ & Viscous friction coefficient & 0.0001 & $\mathrm{~N} \cdot \mathrm{m} \cdot \mathrm{s} / \mathrm{rad}$ \\
\hline
\end{tabular}

Based on the gain tuning procedure described in Section $\mathrm{IV}$, the control gains are chosen as $Q_{0}=\operatorname{diag}(0.1,5,60), R=$ $10^{-4}$ xeye $(2), m_{i}=0.9$, and $n_{i}=0.2$. It should be noted that the system parameters continuously change due to the magnetic saturation and temperature variations during operating time. According to [12], [40] about the variations of the electrical parameters, the stator resistance increases up to $40 \%$ as the temperature in the stator winding increases. In addition, the $q$-axis stator inductance decreases as the stator current increases while the variation of the $d$-axis stator inductance is negligible. On the other hand, the mechanical parameters of the system may extremely vary as the external mechanical load changes [41]. Therefore, to verify the robustness of the proposed nonlinear optimal DTC scheme, it is assumed that the variations of the stator resistance, $q$-axis inductance, equivalent rotor inertia, and viscous friction are selected as $+50 \%,-20 \%,+200 \%$, and $+100 \%$ of the nominal values, respectively. Note that all experimental scenarios are carried out under these parameter variations and the way to change the motor parameters in experiment is explained in detail in [42].

In this paper, the feasibility of the proposed DTC scheme is validated under the following three scenarios:

- Scenario 1 (Low speed region): $\omega_{\text {ref }}=10.5 \mathrm{rad} / \mathrm{s}, T_{L}=$
$0.5 \mathrm{~N} \cdot \mathrm{m}$.

- Scenario 2 (Speed reference change): $\omega_{\text {ref }}=167.6$ $\mathrm{rad} / \mathrm{s} \rightarrow 83.8 \mathrm{rad} / \mathrm{s}, T_{L}=1 \mathrm{~N} \cdot \mathrm{m}$.

- Scenario 3 (Load torque change): $\omega_{\text {ref }}=210 \mathrm{rad} / \mathrm{s}, T_{L}$ $=1 \mathrm{~N} \cdot \mathrm{m} \rightarrow 0 \mathrm{~N} \cdot \mathrm{m}$.

It is noted that these scenarios fully describe the tough operating conditions of ac motor drives in constant torque region.

For a comparative study, the conventional SVM-based PI DTC scheme [11], [25]-[27], [43], [44] is also performed under the same conditions as the proposed SVM-based DTC scheme. With the tuning rules presented in aforementioned references, the bandwidths of the speed, torque, and flux loops are selected as $2 \pi \times 0.3,2 \pi \times 300$, and $2 \pi \times 1200 \mathrm{rad} / \mathrm{s}$, respectively.

\section{B. Results and Discussions}

Figs. 4 to 6 show the experimental results of the proposed nonlinear optimal DTC scheme and conventional PI DTC scheme under Scenarios 1, 2, and 3, respectively. That is, Figs. 4(a) to 6(a) depict the experimental results of the proposed DTC scheme, while Figs. 4(b) to 6(b) show the experimental results of the conventional PI DTC scheme. Each plot shows the waveforms of the speed reference $\left(\omega_{\text {ref }}\right)$, measured speed $(\omega)$, electromagnetic torque $\left(T_{e}\right)$, and stator flux linkage $\left(\lambda_{s}\right)$.

Fig. 4 demonstrates the performances of the proposed DTC method and the conventional PI DTC method at low speed range (i.e., $2 \%$ of the rated speed). As depicted in Fig. 4 , the speed and torque waveforms of the conventional PI DTC scheme (i.e., $3.2 \mathrm{rad} / \mathrm{s}, 0.5 \mathrm{~N} \cdot \mathrm{m}$ ) contain more ripples than those of the proposed DTC scheme (i.e., $0.6 \mathrm{rad} / \mathrm{s}, 0.3$ $\mathrm{N} \cdot \mathrm{m})$. It should be noticed that the speed response of the conventional PI DTC method is not so stable (e.g., frequent undershoots) as that of the proposed DTC method.

Fig. 5 illustrates the experimental results of the proposed DTC scheme and the conventional PI DTC scheme when the speed reference $\left(\omega_{r e f}\right)$ changes. As shown in the figure, the speed response of the former method $(58 \mathrm{~ms})$ is much faster than that of the latter method $(70 \mathrm{~ms})$. Besides, the speed and torque ripples of the proposed DTC scheme $(8.4 \mathrm{rad} / \mathrm{s}, 0.3$ $\mathrm{N} \cdot \mathrm{m}$ ) are smaller than those of the conventional DTC scheme (13.3 rad/s, $0.7 \mathrm{~N} \cdot \mathrm{m})$.

Fig. 6 presents the experimental results of the proposed DTC scheme and the conventional PI DTC scheme when the load torque $\left(T_{L}\right)$ changes. It is clear that the electromagnetic torque $\left(T_{e}\right)$ response of the conventional method $(75 \mathrm{~ms})$ is slower than that of the proposed method $(60 \mathrm{~ms})$. Moreover, the proposed DTC technique can remarkably reduce the speed and torque ripples as compared to those of the conventional method (Proposed: $0.8 \mathrm{rad} / \mathrm{s}, 0.2 \mathrm{~N} \cdot \mathrm{m}$; Conventional: $4.2 \mathrm{rad} / \mathrm{s}, 0.45 \mathrm{~N} \cdot \mathrm{m}$ ). Based on Figs. 4-6, Table II summarizes the comparative performance details of the proposed DTC scheme and the conventional PI DTC 
scheme.

TABLE II

COMPARISON BETWEEN TWO DTC SCHEMES IN EXPERIMENTAL STUDIES

\begin{tabular}{|c|l|c|c|}
\hline Scenario & Criteria & $\begin{array}{c}\text { Proposed DTC } \\
\text { scheme }\end{array}$ & $\begin{array}{c}\text { Conventional PI } \\
\text { DTC scheme }\end{array}$ \\
\hline \multirow{2}{*}{1} & Speed ripple $(\mathrm{rad} / \mathrm{s})$ & 0.6 & 3.2 \\
\cline { 2 - 4 } & Torque ripple $(\mathrm{N} \cdot \mathrm{m})$ & 0.3 & 0.5 \\
\hline
\end{tabular}

\begin{tabular}{|c|l|c|c|}
\hline \multirow{3}{*}{2} & Settling time of speed $(\mathrm{ms})$ & 58 & 70 \\
\cline { 2 - 4 } & Speed ripple $(\mathrm{rad} / \mathrm{s})$ & 8.4 & 13.3 \\
\cline { 2 - 4 } & Torque ripple $(\mathrm{N} \cdot \mathrm{m})$ & 0.3 & 0.7 \\
\hline \multirow{3}{*}{3} & Settling time of torque $(\mathrm{ms})$ & 60 & 75 \\
\cline { 2 - 4 } & Speed ripple $(\mathrm{rad} / \mathrm{s})$ & 0.8 & 4.2 \\
\cline { 2 - 4 } & Torque ripple $(\mathrm{N} \cdot \mathrm{m})$ & 0.2 & 0.45 \\
\hline
\end{tabular}

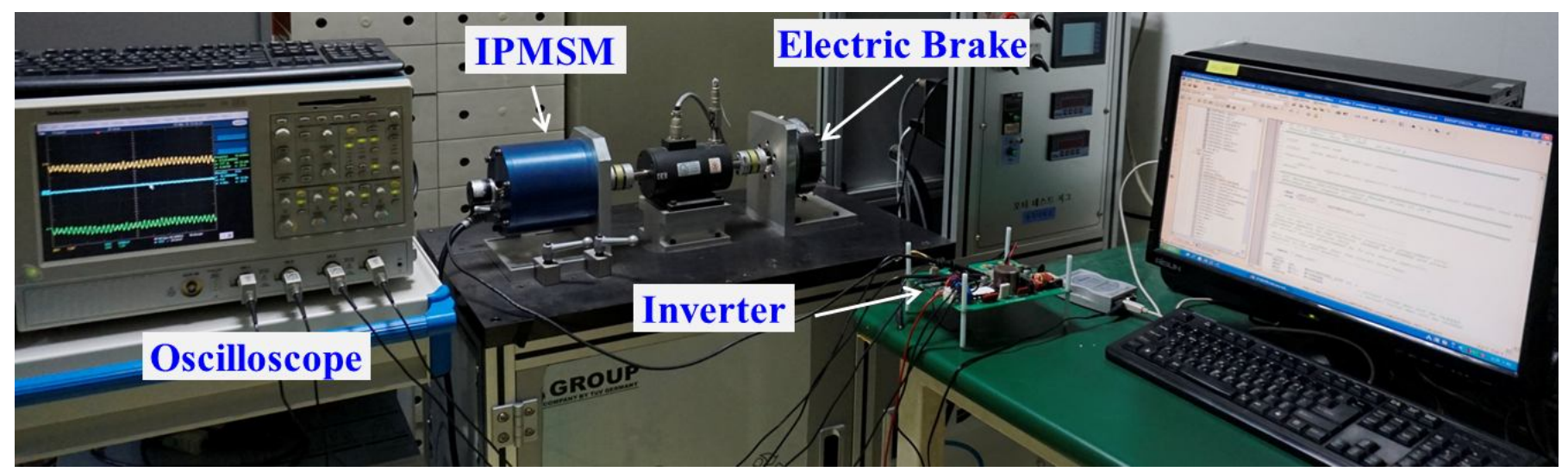

Fig. 3. Experimental setup of the prototype IPMSM drive system.

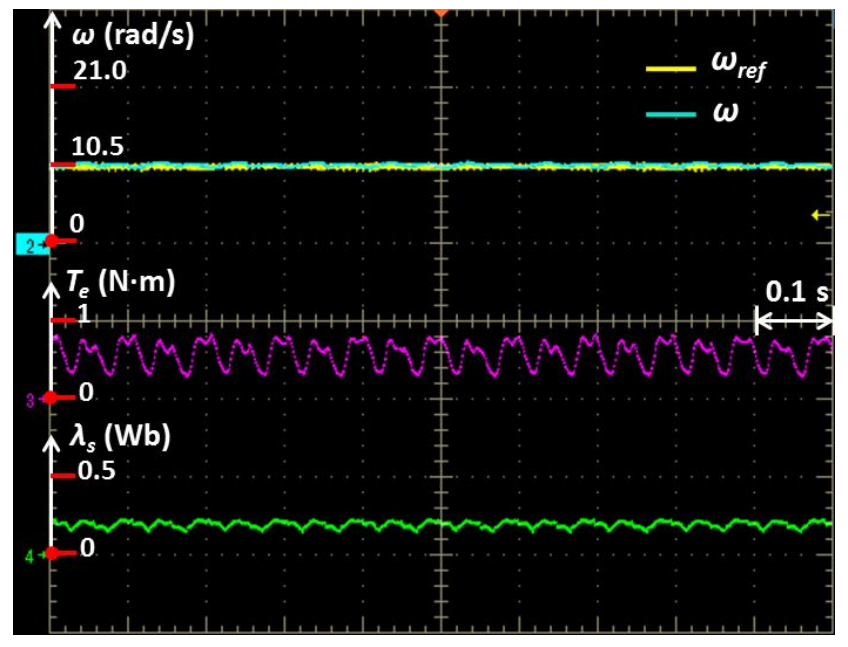

(a)

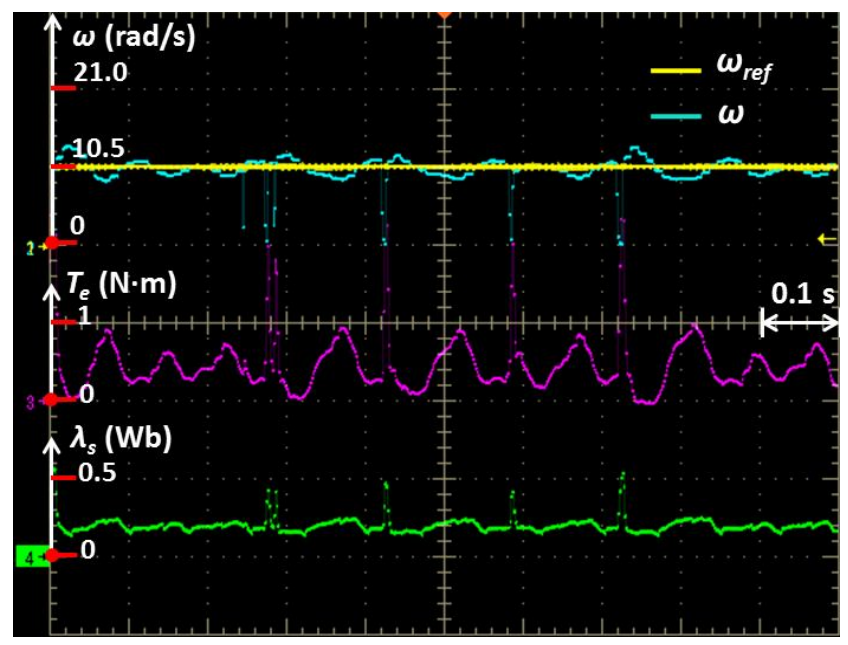

(b)

Fig. 4. Experimental results at low speed region. (a) The proposed nonlinear optimal DTC scheme. (b) The conventional PI DTC scheme. 


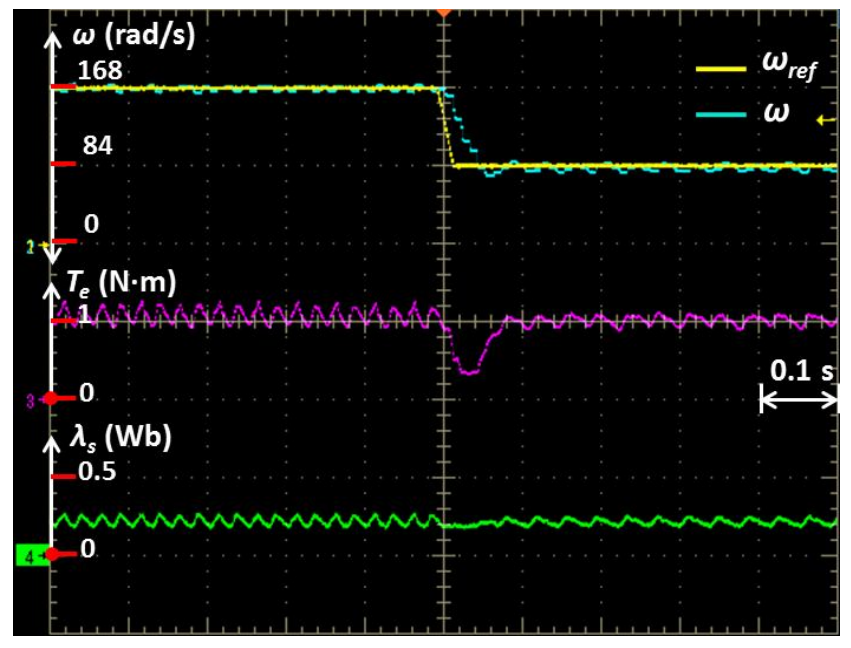

(a)

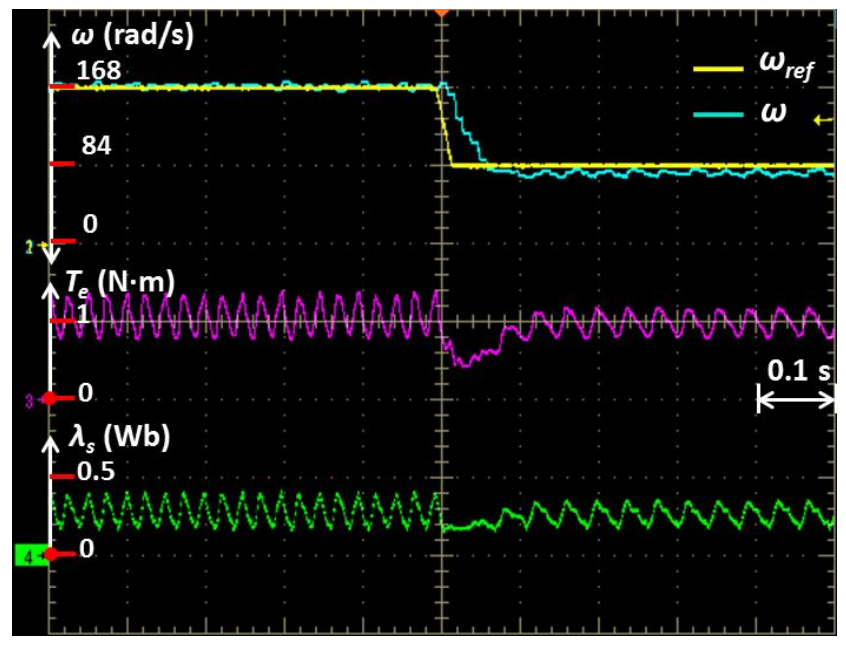

(b)

Fig. 5. Experimental results at speed reference change. (a) The proposed nonlinear optimal DTC scheme. (b) The conventional PI DTC scheme.

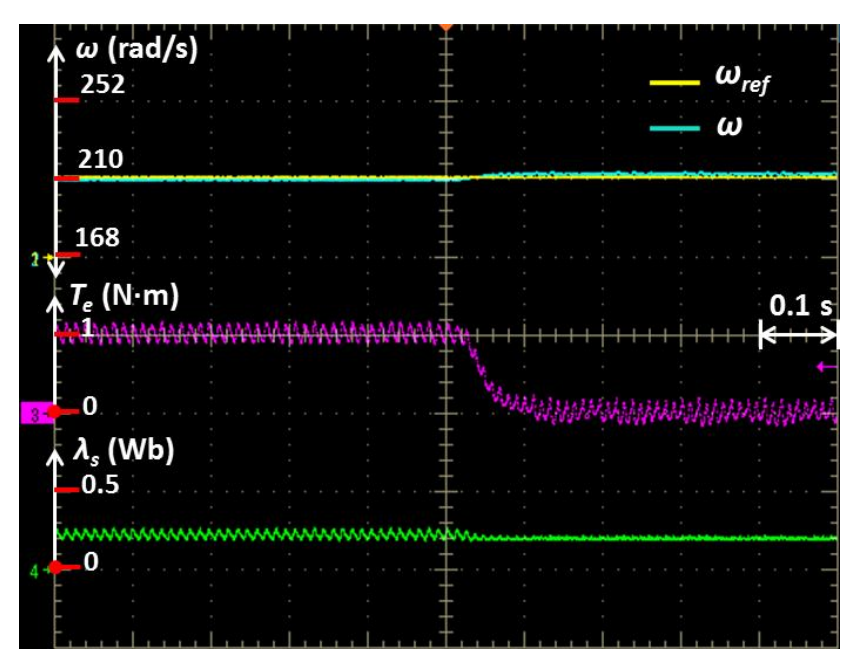

(a)

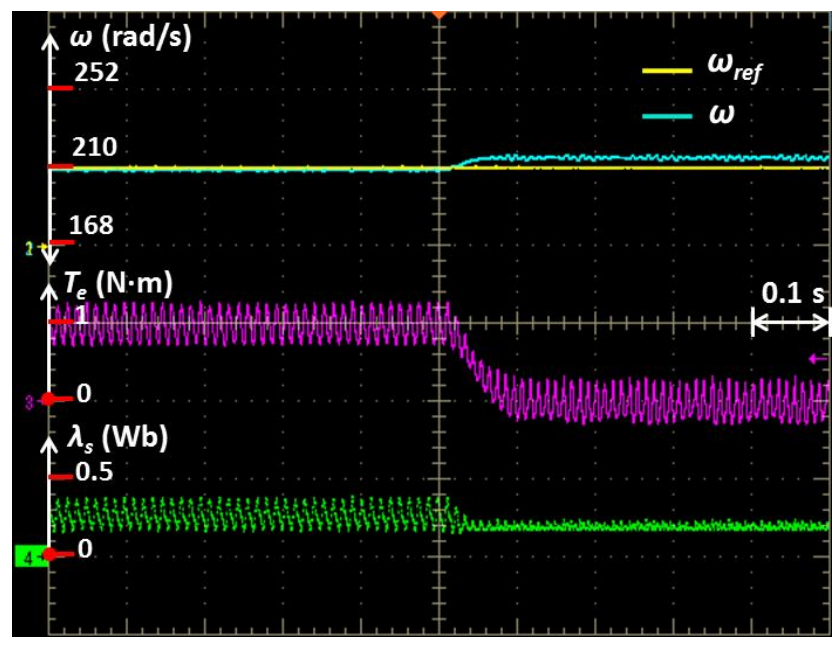

(b)

Fig. 6. Experimental results at load torque change. (a) The proposed nonlinear optimal DTC scheme. (b) The conventional PI DTC scheme.

\section{CONCLUSION AND FUTURE WORK}

This paper suggested a nonlinear optimal DTC technique of IPMSM traction drives based on an offline approximation approach for EV applications. Because of being constructed in the stationary reference frame, the proposed DTC scheme did not need the stator flux angle information which is essential in the conventional PI DTC scheme. Therefore, the proposed method is much simpler and more robust than the conventional PI DTC method because it eliminates Park's transformation and is independent of the estimated stator flux angle that is not accurate in low speed range. In addition, the nonlinearity of the system was effectively dealt with by an approximated nonlinear optimal control law. To verify the effectiveness and feasibility of the proposed DTC strategy, experiments were carried out on a prototype IPMSM drive using a TMS320F28335 DSP. The experimental results under a full set of scenarios showed that the proposed nonlinear optimal DTC method could significantly improve the control performances of the conventional PI DTC scheme in terms of robustness at low speed region, fast transient response time at speed reference and load torque changes, and small speed and torque ripples.

Although the proposed technique was tested on a typical IPMSM drive in constant torque region, the design was presented in a general form, which is applicable to other types of the IPMSM drives in different applications. In the future work, the proposed control algorithm will be extended to the flux-weakening control region with maximum torque per voltage (MTPV) operation of the IPMSM drives.

\section{REFERENCES}

[1] B. M. Baumann, G. Washington, B. C. Glenn, and G. Rizzoni, "Mechatronic design and control of hybrid electric vehicles," IEEE/ASME Trans. Mechatronics, vol. 5, no. 1, pp. 58-72, Mar. 2000.

[2] G. Rizzoni, L. Guzzella, and B. M. Baumann, "Unified modelling of hybrid electric vehicle drivetrains," IEEE/ASME Trans. Mechatronics, vol. 4, no. 3, pp. 246-257, Sep. 1999.

[3] M. A. Rahman and R. Qin, "A permanent magnet hysteresis hybrid synchronous motor for electric vehicles," IEEE Trans. Ind. Electron., vol 
44, no. 1, pp. 46-53, Feb. 1997.

[4] A. Raisemche, M. Boukhnifer, C. Larouci, and D. Diallo, "Two active fault tolerant control schemes of induction motor drive in EV or HEV," IEEE Trans. Veh. Technol., IEEE Trans. Veh. Technol., vol. 63, no. 1, pp. 19-29, Jan. 2014.

[5] B. Tabbache, A. Kheloui, and M. E. H. Benbouzid, "An adaptive electric differential for electric vehicles motion stabilization," IEEE Trans. Veh. Technol., vol. 60, no. 1, pp. 104-110, Jan. 2011.

[6] B. Tabbache, M. E. H. Benbouzid, A. Kheloui, and J. M. Bourgeot, "Virtual-sensor-based maximum-likelihood voting approach for faulttolerant control of electric vehicle powertrains," IEEE Trans. Veh. Technol., vol. 62, no. 3, pp. 1075-1083, Mar. 2013.

[7] J. Yu, W. Pei, and C. Zhang, "A loss-minimization port-controlled Hamilton scheme of induction motor for electric vehicles," IEEE/ASME Trans. Mechatronics, in press.

[8] L. Parsa and H. A. Toliyat, "Fault-tolerant interior-permanent-magnet machines for hybrid electric vehicle applications," IEEE Trans. Veh. Technol., vol. 56, no. 4, pp. 1546-1552, Jul. 2007.

[9] Y. S. Jeong, S. K. Sul, S. E. Schulz, and N. R. Patel, "Fault detection and fault-tolerant control of interior permanent-magnet motor drive system for electric vehicle" IEEE Trans. Ind. Appl., vol. 41, no. 1, pp. 46-51, Jan./Feb. 2005

[10] A. A. M. Hilairet and D. Diallo, "Design of a fault-tolerant controller based on observer for a PMSM drive," IEEE Trans. Ind. Electron., vol. 58, no. 4, pp. 1416-1427, Apr. 2011.

[11] G. F. H. Beng, Sensorless direct torque and flux control of interior permanent magnet synchronous motors at very low speeds including standstill, Ph. D. Thesis, The University of New South Wales, Australia, 2010.

[12] O. Wallmark, On Control of Permanent-Magnet Synchronous Motors in Hybrid-Electric Vehicle Application, Licentiate of Engineering Thesis, Chalmers University of Technology, Sweden, 2004.

[13] H. Rehman and L. Xu, "Alternative energy vehicles drive system: control, flux and torque estimation, and efficiency optimization," IEEE Trans. Veh. Technol., vol. 60, no. 8, pp. 3625-3634, Oct. 2011.

[14] M. F. Rahman, L. Zhong, and K. W. Lim "A direct torque-controlled interior permanent magnet synchronous motor drive incorporating field weakening," IEEE Trans. Ind. Appl., vol. 34, no. 6, pp. 1246-1253, Nov./Dec. 1998.

[15] I. Takahashi and T. Noguchi, "A new quick-response and high-efficiency control strategy of an induction motor," IEEE Trans. Ind. Appl., vol. IA22, no. 5, pp. 820-827, Sep. 1986.

[16] L. Zhong, M. F. Rahman, W. Y. Hu, and K. W. Lim, "Analysis of direct torque control in permanent magnet synchronous motor drives," IEEE Trans. Power Electron., vol. 12, no. 3, pp. 528-536, May 1997.

[17] K. Jezernik, J. Korelic, and R. Horvat, "PMSM sliding mode FPGA-based control for torque ripple reduction," IEEE Trans. Power Electron., vol. 28, no. 7, pp. 3549-3556, Jul. 2013.

[18] C. Xia, J. Zhao, Y. Yan, and T. Shi, "A novel direct torque control of matrix converter-fed PMSM drives using duty cycle control for torque ripple reduction," IEEE Trans. Ind. Electron., vol. 61, no. 6, pp. 27002713, Jun. 2014.

[19] C. Ortega, A. Arias, C. Caruana, J. Balcells, and C. M. Asher, "Improved waveform quality in the direct torque control of matrix-converter-fed PMSM drives," IEEE Trans. Ind. Electron., vol. 57, no. 6, pp. 21012110, Jun. 2010.

[20] S. Mathapati and J. Bocker, "Analytical and offline approach to select optimal hysteresis bands of DTC for PMSM," IEEE Trans. Ind. Electron., vol. 60, no. 3, pp. 885-895, Mar. 2013.

[21] Y. Zhang and J. Zhu, "A novel duty cycle control strategy to reduce both torque and flux ripples for DTC of permanent magnet synchronous motor drives with switching frequency reduction," IEEE Trans. Power Electron., vol. 26, no. 10, pp. 3055-3067, Oct. 2011.

[22] Y. Zhang and J. Zhu, "Direct torque control of permanent magnet synchronous motor with reduced torque ripple and commutation frequency," IEEE Trans. Power Electron., vol. 26, no. 1, pp. 235-248, Jan. 2011.

[23] M. Preindl and S. Bolognani, "Model predictive direct torque control with finite control set for PMSM drive systems, Part 1: Maximum torque per ampere operation," IEEE Trans. Ind. Inform., vol. 9, no. 4, pp. 19121921, Nov. 2013.
[24] H. Zhu, X. Xiao, and Y. Li, "Torque ripple reduction of the torque predictive control scheme for permanent-magnet synchronous motors," IEEE Trans. Ind. Electron., vol. 59, no. 2, pp. 871-877, Feb. 2012.

[25] Y. A. R. I. Mohamed, "Direct instantaneous torque control in direct drive permanent magnet synchronous motors-a new approach," IEEE Trans. Energy Convers. vol. 22, no. 4, pp. 829-838, Dec. 2007.

[26] Z. Xu and M. F. Rahman, "Comparison of a sliding observer and a Kalman filter for direct-torque-controlled IPM synchronous motor drives," IEEE Trans. Ind. Electron., vol. 59, no. 11, pp. 4179-4188, Nov. 2012.

[27] G. Foo and M. F. Rahman, "Sensorless direct torque and flux-controlled IPM synchronous motor drive at very low speed without signal injection," IEEE Trans. Ind. Electron., vol. 57, no. 1, pp. 395-403, Jan. 2010.

[28] K. Gulez, A. A. Adam, and H. Pastaci, "A novel direct torque control algorithms for IPMSM with minimum harmonics and torque ripples," IEEE/ASME Trans. Mechatronics, vol. 12, no. 2, pp. 223-227, Apr. 2007.

[29] M. Xin and S. N. Balakrishnan, "A new method for suboptimal control of a class of non-linear systems," Opt. Contr. Appl. Meth., vol. 26, no. 2, pp. 55-83, Mar./Apr. 2005.

[30] M. Xin, S. N. Balakrishnan, and E. J. Ohlmeyer, "Integrated guidance and control of missiles with $\theta-D$ method," IEEE Trans. Contr. Syst. Tech., vol. 14, no. 6, pp. 981-992, Nov. 2006.

[31] T. D. Do, H. H. Choi, and J. W. Jung, " $\theta-D$ approximation technique for nonlinear optimal speed control design of surface-mounted PMSM drives," IEEE/ASME Trans. Mechatronics, in press.

[32] T. D. Do, H. H. Choi, and J. W. Jung, "SDRE-based near optimal control system design for PM synchronous motor," IEEE Trans. Ind. Electron., vol. 59, no. 11, pp. 4063-4074, Nov. 2012.

[33] T. D. Do, S. Kwak, H. H. Choi, and J. W. Jung, "Suboptimal control scheme design for interior permanent magnet synchronous motors: An SDRE-based approach," IEEE Trans. Power Electron., vol. 29, no. 6, pp. 3020-3031, Jun. 2014.

[34] M. N. Uddin, M. A. Abido, and M. A. Rahman, "Development and implementation of a hybrid intelligent controller for interior permanentmagnet synchronous motor drives," IEEE Trans. Ind. Appl., vol. 40, no. 1, pp. 68-76, Jan./Feb. 2004.

[35] R. S. Rebeiro and M. N. Uddin, "Performance analysis of an FLC-based online adaptation of both hysteresis and PI controllers for IPMSM drive," IEEE Trans. Ind. Appl., vol. 48, no. 1, pp. 12-19, Jan./Feb. 2012.

[36] H. H. Choi, N. T. T. Vu, and J. W. Jung, "Digital implementation of an adaptive speed regulator for a PMSM," IEEE Trans. Power Electron., vol. 26, no. 1, pp. 3-8, Jan. 2011.

[37] J. Astrom and B. Witternmark, Computer-Controlled Systems - Theory and Design, Englewood Cliffs, New Jersey: Prentice-Hall, 1990.

[38] O. Vainio, "Adaptive derivative estimation for delay-constrained acceleration measurement," IEEE Trans. Ind. Electron., vol. 46, no. 5, pp. 933-935, Oct. 1999.

[39] C. F. Lin, Advanced Control Systems Design, Englewood Cliffs, New Jersey: Prentice-Hall Inc., 1994.

[40] R. Monajemy, Control Strategies and Parameter Compensation for Permanent Magnet Synchronous Motor Drives, Ph.D. Dissertation, Virginia Polytechnic Institute and State University, 2000.

[41] H. Huang and L. Chang, "Electrical two-speed propulsion by motor winding switching and its control strategies for electric vehicles," IEEE Trans. Veh. Technol., vol. 48, no. 2, pp. 607-618, Mar. 1999.

[42] N. T. T. Vu, D. Y. Yu, H. H. Choi, and J. W. Jung, "T-S fuzzy modelbased sliding mode control for surface-mounted permanent magnet synchronous motors considering uncertainties," IEEE Trans. Ind. Electron., vol. 60, no. 10, pp. 4281-4291, Oct. 2013.

[43] G. Pellegrino, E. Armando, and P. Guglielmi, "Direct flux field-oriented control of IPM drives with variable DC link in the field-weakening region" IEEE Trans. Ind. Appl., vol. 45, no. 5, pp. 1619-1627, Sep./Oct. 2009.

[44] T. D. Nguyen, G. Foo, K. J. Tseng, and D.M. Vilathgamuwa, "Modeling and sensorless direct torque and flux control of a dual-airgap axial flux permanent-magnet machine with field-weakening operation," IEEE/ASME Trans. Mechatronics, vol. 19, no. 2, pp. 412-422, Apr. 2014.

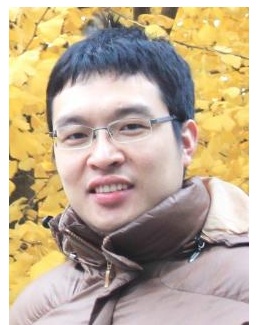

Ton Duc Do (S'12-M'14) received the B.S. and M.S degrees in electrical engineering from Hanoi University of Science and Technology (HUST) in 2007 and 2009, 
respectively, and the Ph.D. degree in the Division of Electronics and Electrical Engineering, Dongguk University, Seoul, Korea, in 2014.

From 2008 to 2009, he worked at the Division of Electrical Engineering, Thuy Loi University, Hanoi, Vietnam, as a lecturer. He also worked at the Division of Electronics and Electrical Engineering, Dongguk University, Seoul, Korea as a postdoctoral researcher. Currently, he is a Senior Researcher at the Pioneer Research Center for Controlling Dementia by Converging Technology, Gyeongsang National University, Jinju, Gyeongsang Nam-do, Korea. His research interests are in the field of advanced control design for electric machine drives, and distributed generation systems using renewable energy sources, and uninterruptible power supplies, electromagnetic actuator system, and targeted drug delivery system.

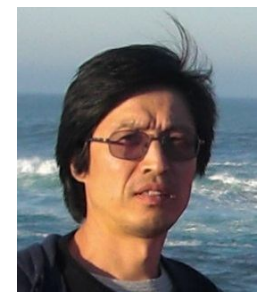

Han Ho Choi (M'03) received the B.S. degree in control and instrumentation engineering from Seoul National University, Seoul, Korea, in 1988, and the M.S. and Ph.D. degrees in electrical engineering from Korea Advanced Institute of Science and Technology (KAIST), Daejeon, Korea, in 1990 and 1994, respectively.

From 1994 to 1998 , he was a Team Leader with the Advanced Technology Laboratory, DaeWoo Electrical Company. He is currently with the Division of Electronics and Electrical Engineering, Dongguk University, Seoul, Korea. He spent his sabbatical with the Department of Electrical and Computer Engineering, California State Polytechnic University, Pomona. He teaches introductory electrical engineering courses on microprocessors, robotics, sensors, and instrumentation engineering. His research interests include linear-matrixinequality-based control system design, microprocessor-based control systems, and variable structure systems.

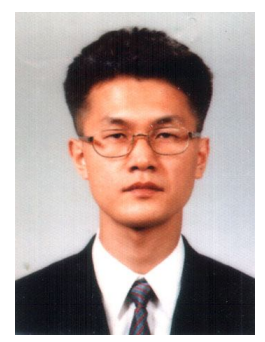

Jin-Woo Jung (S'97-M'06) received the B.S. and M.S. degrees in electrical engineering from Hanyang University, Seoul, Korea in 1991 and 1997, respectively, and the Ph.D. degree in Electrical and Computer Engineering from The Ohio State University, Columbus, Ohio, USA, in 2005.

From 1997 to 2000, he was with the Digital Appliance Research Laboratory, LG Electronics Co., Ltd., Seoul, Korea. From 2005 to 2008, he worked as a Senior Research Engineer at the R\&D Center and with the PDP Development Team, Samsung SDI Co., Ltd., Yongin, Korea. Since 2008, he has been an Associate Professor with the Division of Electronics and Electrical Engineering, Dongguk University, Seoul, Korea. His current research interests include DSP-based electric machine drives, distributed generation systems using renewable energy sources, and power conversion systems and drives for electric vehicles (EVs). 